\title{
Antimalarial in dermatology: 62 cases
}

\author{
A Lahlou*, S Elloudi, H Baybay, and FZ Mernissi \\ University Hospital Hassan II, Dermatology Department, Fez, Morocco
}

\begin{abstract}
Although chloroquine, hydroxychloroquine and quinacrine were originally developed for the treatment of malaria, these medications have been used to treat skin disease for over 50 years. Recent clinical data have confirmed the usefulness of these medications for the treatment of lupus erythematosus. through the study of 62 patients under antimalarial followed in dermatology, we try to clarify the different antimalarial used within dermatology through their mechanism of action and mechanism of action, as well as indicating their clinical uses. In addition, contraindications, adverse effects, of antimalarial are reviewed.
\end{abstract}

\section{Introduction}

The antimalarial, derive from the quinolizine core of quinine. Their initial therapeutic indication was the treatment of malaria attacks but, because of anti-inflammatory and immuno-modulatory activities, they have been since used to treat many other pathologies, in particularly dermatological ones. We provide a series of 62 cases to discuss their use in dermatology, indications, side effects and Evolution under treatment.

\section{Material and methods}

We retrospectively reviewed the clinical records of all patients who are under antimalarial in dermatology department at University Hospital HASSAN II Fez during the period from 2011 to 2016, to identify indications and therapeutic modalities.

\section{Results}

62 patients were collected, the mean age was 41 years old, sex ratio $\mathrm{F} / \mathrm{H}=3$; although $13,4 \%$ of patients had a history of autoimmune disease, The antimalarial were administered to $25 \%$ of lupus that was chronic in $30 \%$ of cases, amigo in $16 \%$ of cases, in to $40 \%$ of dermatomyositis, and $12 \%$ of sarcoidosis , $43 \%$ of patients had systemic signs, $33 \%$ of photosensitivity, $25 \%$ arthralgia, $11 \%$, dry syndrome, 95\% had a skin disease which had improved under antimalarial, a pretherapeutic was practiced among all the patients who came back normal in $90 \%$ of cases, in addition $41 \%, 41 \%$ of patients were put under hydroxychloroquine despite its non-availability in Morocco, $59 \%$ under chloroquine for a mean period of 27 months and a mean dose of $263 \mathrm{mg} /$ day. $22 \%$ of patients had side effects, $30 \%$ of pigmentary disorders, 2 cases of rash, 6 cases had presented a visual field, and 3 cases of retinopathy, $62 \%$ of patients were on combination treatment with a favorable evolution de $69 \%$ of cases.

\section{Discussion}

Antimalarial drugs have been in common use in dermatology [1]. Their mechanism of action is complex, and it is now known that they act through various pathways, and have immunomodulatory action, anti-inflammatory, antiproliferative, and photoprotective effects [2]. Antimalarial are effective for the treatment of the specific skin lesions of cutaneous Lupus erythematosus, whether acute, subacute, or chronic, the beneficial effects of quinine in patients with lupus erythematosus (LE) were published in 1894 by Payne, who reported the successful treatment of discoid LE with this drug [3]. First synthetic antimalarial, quinacrine (QC), was made in1930. Chloroquine (CQ) and hydroxychloroquine (HCQ) fool-lowed (CQ was first synthesized in 1934 and HCQ in 1955) [4]. The use of antimalarial as a treatment for Lupus erythematosus became widespread in 1951, with the publication of an article by which described good response to QC treatment in 18 patients with LE [5]. They are also used for treatment of dermatomyositis, Sarcoidosis [6,7] and others such us Late cutaneous porphyria and chronic ulcerative stomatitis, or lichen planus [8] in our context they are used mainly for lupus, dermatomyositis and sarcoidosis.

They have many side effects dominated by the irreversible ocular involvement [9] imposing an ophthalmologic examination, color and visual field test, prior to treatment initiation and an annual monitoring.

\section{Conclusion}

Antimalarial drugs had an effective treatment that can be safe for the patient and can cause secondary effects of the interest or irreversible a pretreatment investigations with Clinical and biological monitoring of patients under treatment

\section{References}

1. Goldman L, Cole DP, Preston RH (1953) Chloroquine phosphate in treatment of discoid lupus erythematosus. JAMA 152: 1428-1429.

2. Kaufmann AM, Krise JP (2007) Lysosomal sequestration of amine-containing drugs: analysis and therapeutic implications. JPharm Sci 96: 729-746.

3. Wallace DJ (1996) The history of antimalarials. Lupus 5: S2-S3.

4. Van Beek MJ, Piette WW (2001) Antimalarials. Dermatol Clin 19: 147-160.

5. Page F (1951) Treatment of lupus erythematosus with mepacrine. Lancet 2: 755-758.

Correspondence to: Asmae Lahlou, Departement of Dermatology, Hospital University, Hassan II, FEZ, Morocco, E-mail: lahlouasmae@gmail.com

Key words: antimalarial drugs, dermatomyositis, lupus erythematosus, sarcoidosis

Received: September 28, 2016; Accepted: October 29, 2016; Published: November 03, 2016 
6. Wollina U, Kostler E, Koch A, Riedel H, Stolzel U (2009) Does chloroquine therapy of porphyria

7. cutanea tarda influence liver pathology? Int J Dermatol 48: 1250-1253.

8. Ang GC, Werth VP (2005) Combination of antimalarials in the treatment of cutaneous dermatomyositis:
9. a retrospective study. Arch Dermatol 141: 855-859

10. Wolf R, Wolf D, Ruocco V (2000) Antimalarials: unapproved uses or indications. Clin Dermatol 18: 17-35.

11. Ruiz-Irastorza G, Ramos-Casals M, Brito-Zeron P, Khamashta MA (2010) Clinica efficacy and side effects of antimalarials in systemic lupus erythematosus: a systematic review. Ann Rheum Dis 69: 20-28.

Copyright: ( 02016 Lahlou A. This is an open-access article distributed under the terms of the Creative Commons Attribution License, which permits unrestricted use, distribution, and reproduction in any medium, provided the original author and source are credited. 